\title{
Designer receptors: therapeutic adjuncts to cell replacement therapy in Parkinson's disease
}

\author{
Elena M. Vazey and Gary Aston-Jones
}

Department of Neurosciences, Medical University of South Carolina, Charleston, South Carolina, USA.

\begin{abstract}
Cell replacement for restoring neuronal populations in Parkinson's disease has been demonstrated as a potential therapeutic strategy over several decades of studies; however, a number of issues regarding sources of replacement neurons and optimization of therapeutic efficacy in vivo have hampered clinical implementation. In this issue of the JCI, Dell'Anno and colleagues evaluated the use of induced dopaminergic (iDA) neurons that were generated by direct fibroblast reprogramming for transplantation and demonstrated that postmitotic iDA neurons stably and functionally integrate into host striatum to produce motor improvements in 6-OHDA rats, a Parkinson's disease model. Furthermore, using designer receptors exclusively activated by designer drugs (DREADDs) in iDA grafts to noninvasively increase dopamine release from grafted neurons, the authors were able to remotely control transplanted neurons and enhance therapeutic efficacy. This initial proof-of-concept study is the first application of DREADD technology to treat neurodegenerative dysfunction, and by using DREADDs as an adjunct to iDA cell therapy, it presents a novel strategy to overcome some current caveats of cell replacement therapy.
\end{abstract}

The loss of nigral dopaminergic (DA) cells in Parkinson's disease (PD) was discovered in the mid-twentieth century (1), and DA cell replacement was quickly posited as a potential therapeutic strategy. By the late 1970s, proof-of-function studies emerged and showed that intrastriatal transplantation of fetal ventral mesenphalic (VM) tissue could survive in the dopamine-depleted striatum and improve motor function deficits in PD animal models (2). Clinical trials with VM tissue and cell suspensions have been undertaken for nearly 30 years; however, there has yet to be an approved clinical cell replacement therapy for PD. These efforts have not been for naught; a number of clinical trials have demonstrated long-term beneficial effects on motor symptoms (10+ years) and provided valuable lessons $(3,4)$. The hope for an effective cell replacement therapy for PD has survived the test of time, and efforts are consistently being revised and improved. However, many questions - primarily over technical optimization - have prevented widespread clinical implementation.

Conflict of interest: The authors have declared that no conflict of interest exists.

Citation for this article: J Clin Invest. 2014; 124(7):2858-2860. doi:10.1172/JCI76833.

\section{iDA neurons as a cell source} for transplantation

For many decades, primary fetal VM tissue has been the gold standard for transplantation therapy in PD; however, ethical and practical considerations, such as standardization of tissue collection and graft preparation, have limited its widespread use. Currently, the majority of efforts are focused on large-scale generation of pluripotent neural progenitor cells as a renewable and consistent source for transplantation. Posttransplantation cell survival, which is quite poor, benefits from grafting the relatively immature neural precursors provided by fetal tissue and pluripotent cells. Unfortunately, the potential for tumor generation is inherent to the use of immature pluripotent cells; however, direct reprogramming of differentiated cells reduces the potential tumorigenicity of transplanted grafts (5). To this end, Dell'Anno et al. directly reprogrammed fibroblasts with doxycycline-inducible expression of a minimal transcription factor cocktail that bypassed the need to generate pluripotent neural stem/progenitor cells. Furthermore, they continued administration of doxycycline in vivo for one week after transplantation, which was sufficient to induce long-term stability of iDA neurons, with no evidence of tumorigenicity or pluripotency (as mea- sured by Ki67 and nestin expression) in the transplanted grafts (6). Direct reprogramming of differentiated cells has the potential not only to generate a standardized and safe cell source for transplantation in all PD patients, but can also be applied to generate autologous transplants in cases of sporadic or drug-induced PD.

Dell'Anno et al. extensively validated the iDA neuronal phenotype in PD animal models and showed anatomical connectivity within host neural networks as well as functional integration, using fluorescent false neurotransmitters and trans-synaptic neural tracers to identify synaptic junctions within host tissue. Additionally, electrochemistry and electrophysiology confirmed dopamine release and synaptic activity from grafted iDA neurons. Behavioral evaluations, including apomorphineinduced rotations and forelimb akinesia, revealed that iDA graft recipients exhibited significant improvements in motor function compared with sham-treated (fibroblast-treated) animals. Unfortunately, the observed improvements in iDA cell-transplanted animals did not meet the level provided by gold-standard VM transplants (6). iDA neurons surpass many technical limitations of VM tissue as a cell source for transplants; however, given the extensive data on VM transplants in humans, any alternate cell source must provide equal or greater benefit to be of translational use. In an attempt to resolve this disparity between iDA and VM transplants, Dell'Anno and colleagues applied designer receptors exclusively activated by designer drugs (DREADDs) technology to optimize activity from grafted iDA transplants. Although the therapeutic application of DREADD technology in PD, including as an adjunct to transplantation therapy, has been postulated (7), it has not been previously validated in preclinical models.

\section{DREADDs as therapeutic adjuncts for cell replacement in PD}

Dell'Anno et al. engineered iDA neurons to express DREADD receptors and by doing so were able to modulate tonic activ- 
ity through application of the DREADD receptor-specific ligand clozapine-Noxide (CNO), which did not interfere with endogenous signaling pathways. DREADD stimulation modulated the physiological activity of iDA neurons in vitro to a level similar to that of DREADDs in primary VM DA neuron cultures. Stimulation of the Gq-coupled DREADD hM3Dq not only increased basal neural activity and extracellular dopamine released from iDA neurons, but in another novel finding, the authors showed that hM3Dq-mediated activation of iDA neurons rapidly increased intracellular dopamine synthesis (6). Thus, activation of DREADD receptors by their specific ligand not only allows for noninvasive manipulation of iDA neuronal function, but provides extended modulation of DA function beyond the acute effects of the ligand. Although iDA grafts did not restore motor performance to the same level as that in VM grafts, following three days of systemic CNO treatment, DREADD-expressing iDAs restored motor function in both rotational asymmetry and the forelimb akinesia test to levels comparable to those of VM grafts (6). Together, these results demonstrate an original and potentially quite powerful application of DREADDs as an adjunct to potentiate transplantation therapy.

Dell'Anno et al. did not report on additional advantages of DREADD-dependent graft modulation, but their results hint at further potential benefits of combining DREADD technology with transplants (6). For example, DREADDs have been applied to modulate neural circuits in a dose-responsive manner (8); therefore, DREADD-type technology could be used to modify graft performance as needed on an individual basis. DREADDs couple only weakly to the arrestin pathway (9), meaning that DREADDs are rarely internalized or undergo desensitization and thus may be useful for chronic therapy. Indeed, the DREADD ligand CNO is orally bioavailable and rapidly activates DREADDexpressing neural populations for sustained time periods after systemic delivery $(8,10)$, increasing potential clinical utility.

This exciting report by Dell'Anno and colleagues (6) elegantly demonstrates the integration of DREADD technology and cell replacement therapy and is an excellent starting point for future explorations of the therapeutic potential of DREADDexpressing cells in PD and other neurological disorders. Initial proof-of-concept studies like the one by Dell'Anno et al. provide impetus for further exploration of the therapeutic potential of designer receptor technology. No doubt, future studies will apply refined receptor-ligand combinations that will be more amenable for application in human populations (11), selective for other signaling mechanisms, or expressed in a cell type-specific manner to extend clinical application.

\section{Moving forward}

Although Dell'Anno et al. (6) provided valuable evidence for the applicability of iDA-DREADD-based technology in PD cell replacement therapy, several additional considerations need to be taken into account to remedy the attrition between trials and clinical therapies for cell replacement. Although direct fibroblast reprogramming avoids pluripotent cell generation, it requires the introduction of multiple transgenes. Further safety procedures and validation will be required to avoid the elicitation of tumorigenicity by random transgene integration in recipient tissues prior to clinical implementation. Additionally, murine fibroblasts have proven to be more amenable to direct reprogramming than human fibroblasts; therefore, optimization of reprogramming transcription factors will be required to generate stable human fibroblast-derived iDA neurons. Notably, the reprogramming factor cocktail (Ascl1, Nurr1, and Lmx1a) used by Dell'Anno and colleagues produced iDA neurons that expressed low levels of the transcription factors FOXA2 and PITX3, which normally regulate DA neuronal development. Compared with nigral (A9) DA neurons, ventral tegmental area (A10) DA neurons express inherently less PITX3 (12); therefore, iDA neurons may have more of an A10 phenotype rather than an $\mathrm{A} 9$ phenotype, which provides the most functional benefit for replacement of lost nigral DA neurons in preclinical investigations (13). The therapeutic implications of transplanting A10-like DA neurons that exhibit an innate resistance to $\mathrm{PD}$ pathology have not been widely explored. Existing clinical trial data indicate that $\alpha$-synuclein pathology can be transmitted to grafted neurons in vivo (14), but it is not known how grafted DA neurons functionally respond to $\mathrm{PD}$-associated pathological insults. Is the ability to control iDA activity through the use of DREADDs or a similar technology sufficient to overcome pathological insult in grafted neurons and com- pensate for ongoing degenerative processes in the host brain?

Although intrastriatal grafts appear to restore local synaptic interactions, they may not be able reestablish the broader network connections lost as the result of nigral DA neuron degeneration or address PD pathology in other systems. For example, can ectopic grafts remediate aberrant basal ganglia oscillatory activity or influence other pathological symptoms in nonDA systems? Using DREADDs to improve therapeutic efficacy beyond intrastriatal transplantation could be tested through their application to systems that contribute to nonmotor dysfunction in PD, including those systems responsible for symptoms, like cognitive dysfunction, that are not addressed by dopamine replacement. Cholinergic and noradrenergic neurons are prime candidates to target for improving nonmotor dysfunction in PD $(15,16)$. Indeed, DREADDs were recently shown to be effective for functional modulation of locus coeruleus noradrenergic neurons, resulting in significant behavioral effects (10). With a dual-targeting approach, the same ligand could positively modulate both transplanted neurons and residual neurons in another affected system to broaden therapeutic efficacy. Alternately, an additional DREADD-ligand pair could be used as concurrent standalone gene therapy in systems responsible for nonmotor symptoms.

Dell'Anno and colleagues have provided an excellent and novel resolution to some of the current roadblocks preventing clinical application of cell replacement therapy for PD. The results from Dell'Anno et al. complement other recent advances in optimizing clinical candidates and standardizing monitoring of transplantation therapy. Taken together, such findings provide the foundation for moving cell replacement therapy closer toward inclusion in the clinical armamentarium for treating PD.

\section{Acknowledgments}

This work was supported in part by the Parkinson's Disease Foundation, the Alzheimer's Association, and PHS grants R21MH099534 and P50DA015369.

Address correspondence to: Elena M. Vazey, Department of Neurosciences, Medical University of South Carolina, 173 Ashley Avenue, BSB 403, Charleston, South Carolina 29401, USA. Phone: 843.792.5289; Fax: 843.792.4223; E-mail: vazey@musc.edu. 
1. Ehringer H, Hornykiewicz O. [Distribution of noradrenaline and dopamine (3-hydroxytyramine) in the human brain and their behavior in diseases of the extrapyramidal system]. Klin Wochenschr. 1960; 38:1236-1239.

2. Perlow MJ, Freed WJ, Hoffer BJ, Seiger A, Olson L, Wyatt RJ. Brain grafts reduce motor abnormalities produced by destruction of nigrostriatal dopamine system. Science. 1979;204(4393):643-647.

3. Cochen V, et al. Transplantation in Parkinson's disease: PET changes correlate with the amount of grafted tissue. Mov Disord. 2003;18(8):928-932.

4. Lindvall O. Developing dopaminergic cell therapy for Parkinson's disease - give up or move forward? Mov Disord. 2013;28(3):268-273.

5. Ben-David U, Benvenisty N. The tumorigenicity of human embryonic and induced pluripotent stem cells. Nat Rev Cancer. 2011;11(4):268-277.

6. Dell'Anno MT, et al. Remote control of induced dopaminergic neurons in parkinsonian rats. J Clin
Invest. 2014;124(7):3215-3229.

7. Vazey EM, Aston-Jones G. New tricks for old dogmas: optogenetic and designer receptor insights for Parkinson's disease. Brain Res. 2013;1511:153-163.

8. Alexander GM, et al. Remote control of neuronal activity in transgenic mice expressing evolved $\mathrm{G}$ protein-coupled receptors. Neuron. 2009;63(1):27-39.

9. Armbruster BN, Li X, Pausch MH, Herlitze S, Roth BL. Evolving the lock to fit the key to create a family of $\mathrm{G}$ protein-coupled receptors potently activated by an inert ligand. Proc Natl Acad Sci U S A. 2007; 104(12):5163-5168

10. Vazey EM, Aston-Jones G. Designer receptor manipulations reveal a role of the locus coeruleus noradrenergic system in isoflurane general anesthesia. Proc Natl Acad Sci U S A. 2014;111(10):3859-3864.

11. Jann MW, Lam YW, Chang WH. Rapid formation of clozapine in guinea-pigs and man following clozapine-N-oxide administration. Arch Int Pharmacodyn Ther. 1994;328(2):243-250.
12. Korotkova TM, Ponomarenko AA, Haas HL, Sergeeva OA. Differential expression of the homeobox gene Pitx3 in midbrain dopaminergic neurons. Eur J Neurosci. 2005;22(6):1287-1293.

13. Grealish S, Jonsson ME, Li M, Kirik D, Bjorklund A, Thompson LH. The A9 dopamine neuron component in grafts of ventral mesencephalon is an important determinant for recovery of motor function in a rat model of Parkinson's disease. Brain. 2010; 133(pt 2):482-495.

14. Li JY, et al. Lewy bodies in grafted neurons in subjects with Parkinson's disease suggest host-to-graft disease propagation. Nat Med. 2008;14(5):501-503.

15. Vazey EM, Aston-Jones G. The emerging role of norepinephrine in cognitive dysfunctions of Parkinson's disease. Front Behav Neurosci. 2012;6:48.

16. Kehagia AA, Barker RA, Robbins TW. Neuropsychological and clinical heterogeneity of cognitive impairment and dementia in patients with Parkinson's disease. Lancet Neurol. 2010;9(12):1200-1213. 